\title{
Electronic Structure and van der Waals Interactions in the Stability and Mobility of Point Defects in Semiconductors
}

\author{
Wang Gao and Alexandre Tkatchenko* \\ Fritz-Haber-Institut der Max-Planck-Gesellschaft, Faradayweg 4-6, 14195, Berlin, Germany
}

(Dated: July 4, 2013)

\begin{abstract}
We study the role of electronic structure (band gaps) and long-range van der Waals interactions on the stability and mobility of point defects in silicon and heavier semiconductors. Density functional theory calculations with hybrid functionals that contain part of Hartree-Fock exchange energy are essential to achieve reasonable description of defect electronic levels, leading to accurate defect formation energies. However, these functionals significantly overestimate the experimental migration barriers. The inclusion of screened vdW interactions further improves the description of defect formation energies, significantly changes the barrier geometries, and brings migration barrier heights into close agreement with experimental values. These results suggest that hybrid functionals with vdW interactions can be successfully used for predictions in broad range of materials where the correct description of both the electronic structure and the long-range electron correlation is essential.
\end{abstract}

Diffusion of defects in semiconductors is a fundamental process of matter transport. Defects are abundant in essentially all real materials and often they significantly modify the electronic, optical, and magnetic properties of solids. For example, the electron spin of donors in Si and vacancy defects in $\mathrm{SiC}$ have been investigated for their possible use as components of quantum devices [1, 2]. Therefore, the study of defects is important from both fundamental and technological points of view.

Here we focus on understanding the interplay between eletronic structure and non-local correlation effects for the fundamental benchmark case of intrinsic point defects in bulk Si and heavier semiconductors. Two kinds of native point defects in $\mathrm{Si}$, self-interstitials and vacancies, have been intensively investigated both experimentally and theoretically. However, the understanding of self-diffusion in Si remains incomplete, despite decades of seminal work on the subject [3-23]. Using secondary ion mass spectrometry (SIMS), two groups obtained identical conclusions that the vacancies mechanism is preferred over the interstitials mechanism in self-diffusion at low temperature while the interstitials mechanism can be dominant at high temperature $[5,6]$. Correspondingly, the diffusion activation energies (sum of the formation energy and migration barrier $H_{\mathrm{A}}=H_{\mathrm{f}}+H_{\mathrm{m}}$ ) were $H_{\mathrm{A}, \mathrm{I}}$ $=4.96 \mathrm{eV}$ and $H_{\mathrm{A}, \mathrm{V}}=4.42 \mathrm{eV}$ proposed by Bracht et al. based on $\mathrm{B}, \mathrm{As}$, and $\mathrm{P}$ diffusion experiments [6], and $H_{\mathrm{A}, \mathrm{I}}=4.95 \mathrm{eV}$ (extracted from the analysis of $\mathrm{Zn}$ diffusion in $\mathrm{Si}$ [8]) and $H_{\mathrm{A}, \mathrm{V}}=3.6 \mathrm{eV}$ proposed by Itoh et al. [5]. In contrast, SIMS experiments by Ural et $a l$., where the activation energy $H_{\mathrm{A}}$ of the interstitials was extracted from $\mathrm{P}$ and $\mathrm{Sb}$ diffusion experiments [9], found that both interstitials and vacancies have comparable contribution to self-diffusion over a wide temperature range $\left(800-1100{ }^{\circ} \mathrm{C}\right)$. The corresponding measured activation energies were $H_{\mathrm{A}, \mathrm{I}}=4.68 \mathrm{eV}$ and $H_{\mathrm{A}, \mathrm{V}}=4.86$ $\mathrm{eV}$ [10]. Recent SIMS experiments by Vaidyanathan et al. indicated that the interstitial mechanism is dominant in the self-diffusion at the lower temperature range of $650-1000{ }^{\circ} \mathrm{C}[13]$.

First-principles calculations have been instrumental in the understanding of point defects in $\mathrm{Si}$ [14-23]; however, the computation of point defect properties is still fraught with difficulties. Density functional theory (DFT) calculations with the local-density approximation (LDA) or generalized gradient approximation (GGA) usually underestimate defect formation energies due to the electron self-interaction error. Furthermore, both LDA and GGA miss the long-range vdW interactions for nonhomogeneous electron densities. Nevertheless, it is remarkable that GGAs often produce fairly good results for migration barrier heights of point defects [24]. Hybrid DFT functionals mitigate the electron self-interaction error, and yield defect formation energies in better agreement with higher-level $G W$ and quantum Monte Carlo (QMC) calculations [17, 18, 21]. However, these functionals still miss the long-range vdW interactions and often overestimate migration barriers of point defects [23]. In principle, accurate defect energetics could be determined by explicit many-electron methods (self-consistent $G W$ or QMC calculations) [16-18, 21]. Unfortunately, the application of $G W$ and QMC is limited to small supercells due to their rather large computational cost. Recently, Bruneval proposed a range-separation scheme treating short-range correlation effects within LDA and long-range with the random-phase approximation (RPA) for the correlation energy (called rs-LDA-RPA in the following) [23]. While rs-LDA-RPA is a promising method, it is still more expensive than DFT calculations, lacks atomic forces at present, overestimates migration barriers, and could be affected by the limitations of the nonself-consistent RPA correlation energy [25].

In this Letter, we study the stability and diffusion of point defects in bulk Si and heavier semiconductors using hybrid DFT calculations including screened long-range van der Waals (vdW) interactions. This approach can be 
efficiently applied to large supercells required for realistic modeling of defects in semiconductors. Furthermore, the hybrid DFT+vdW method allows us to accurately describe both the electronic properties and the structures of semiconductors on equal footing [26, 27]. Specifically, we use the Heyd-Scuseria-Ernzerhof functional [28], coupled with the recently developed method for screened long-range vdW interactions [29]. The inclusion of vdW interactions has been shown to consistently improve the lattice constants, cohesive energies, and bulk moduli for ionic and semiconductor solids over standard DFT functionals $[27,30]$. The accurate treatment of electrodynamic screening in the DFT+vdW approach is crucial for both pristine and defect-containing semiconductors, as it reduces the vdW $C_{6}$ coefficients for "atoms" in semiconductors by a factor of 1.6 to 1.8 [29], and yields results in agreement with experiments and explicit timedependent DFT (TDDFT) calculations [27]. We find that $\mathrm{HSE}+\mathrm{vdW}$ solves the underestimation of PBE on defect formation energies and the overestimation of HSE on defect migration barriers, thereby yielding both accurate formation energies and migration barriers. For multi-atom vacancies in $\mathrm{Si}$, and point defects in heavier and more polarizable semiconductors (such as Ge, GaAs, InP, and InAs), vdW interactions are shown to play an increasingly larger role on their stabilities.

We mainly focus on neutral point defects in this Letter: split- $\langle 110\rangle(\mathrm{X})$, hexagonal $(\mathrm{H})$, tetrahedral $(\mathrm{T})$, and monovacancy $(\mathrm{V})$, because the neutral defects are dominant under intrinsic doping conditions [31, 32]. All total energy calculations were performed using the FHIaims all-electron code with "tight" computational settings [33]. For hybrid functional calculations, we used an efficient resolution-of-the-identity implementation for the computation of four-center integrals [34]. For comparison purpose, the standard Perdew-Burke-Ernzerhof (PBE) [35] and HSE functionals were employed, as well as the PBE+vdW and HSE+vdW functionals [36]. Our careful convergence tests demonstrate that the interstitials (including vdW interactions) converge very well with 64 atoms supercell, while vacancies require cells with 216 atoms due to the presence of Jahn-Teller (JT) distortion. This finding is in agreement with previous work [23]. We adopt a $k$-point grid of $4 \times 4 \times 4(2 \times 2 \times 2)$ for 64 (216) atom supercells.

The results of our calculations for the formation energy of four point defects in Si are shown in Table I, where comparisons are made with other calculations in the literature and with experimental data. Due to the electron self-interaction error, the PBE functional underestimates the formation energy of interstitials by about $0.7 \mathrm{eV}$ relative to the HSE functional. Both PBE and HSE functionals predict that the $\mathrm{X}$ and $\mathrm{H}$ interstitials have similar stability and are $0.15-0.36 \mathrm{eV}$ more stable than the $\mathrm{T}$ interstitial. On the other hand, the tetrahedric configuration of the vacancy is about $0.43 \mathrm{eV}(0.15 \mathrm{eV})$ less
TABLE I. Formation energies of interstitials (X, H, T) and vacancy $(\mathrm{V})$ point defects with different theoretical methods along with experimental estimates.

\begin{tabular}{ccccc}
\hline \hline & $\mathrm{X}$ & $\mathrm{H}$ & $\mathrm{T}$ & $\mathrm{V}$ \\
\hline PBE & 3.69 & 3.75 & 3.90 & 3.58 \\
PBE+vdW & 3.75 & 3.73 & 3.75 & 3.65 \\
HSE & 4.43 & 4.49 & 4.74 & 4.19 \\
HSE [23] & 4.40 & 4.52 & & \\
HSE+vdW & 4.41 & 4.40 & 4.51 & 4.38 \\
rs-LDA-RPA [23] & 4.49 & 4.74 & & 4.33 \\
GW [21] & 4.40 & 4.46 & 4.51 & \\
QMC [17] & 4.94 & 5.05 & 5.13 & \\
Exp [3-5, 7-12] & & $4.2-4.7$ & & $2.1-$ \\
& & & & \\
\hline \hline
\end{tabular}

stable than the $\mathrm{X}$ interstitials when using the HSE (PBE) functional. Once the vacancy configuration is relaxed, it experiences the JT distortion, and becomes more stable than the $\mathrm{X}$ interstitial, having formation energy $H_{\mathrm{f}, \mathrm{PBE}}$ $=3.58 \mathrm{eV}$ and $H_{\mathrm{f}, \mathrm{HSE}}=4.19 \mathrm{eV}$. The above results are consistent with previous PBE, HSE, and rs-LDA-RPA studies in the literature [16-18, 21, 23, 37].

In contrast to the well-known role of exact exchange, the influence of long-range vdW interactions on defect formation energies has not been assessed before. vdW interactions are part of the long-range electron correlation energy and they are missing from all semi-local and hybrid functionals. The influence of vdW interactions on binding energies of molecular systems has been studied in much detail, but the role of vdW interactions on cohesion in semiconductor and metallic solids is still under debate [38]. Even in covalently-bound semiconductors, such as Si and Ge, the long-range vdW energy has been found to contribute around $0.2 \mathrm{eV}$ per atom [27]. Here we study the effect of vdW interactions on the defect formation energy, focusing on the HSE and HSE+vdW results in Table I. The vdW interactions only slightly change the structures of interstitials and vacancies, increasing the distance between the defect atom and the surrounding atoms by less than $0.02 \AA$. In the case of interstitials, $\mathrm{vdW}$ interactions decrease the formation energy of $\mathrm{X}, \mathrm{H}$, and $\mathrm{T}$ by $0.02,0.09$, and $0.23 \mathrm{eV}$, respectively. Consequently, the energy difference between the $\mathrm{X}$ and $\mathrm{T}$ defects is decreased from $0.31 \mathrm{eV}$ (by HSE) to $0.10 \mathrm{eV}$ (by HSE+vdW). Thus, the three interstitials are likely to coexist in Si due to the effect of vdW interactions. The vdW interactions reduce the difference between $\mathrm{T}$ and $\mathrm{V}$ defects from $0.55 \mathrm{eV}$ to $0.13 \mathrm{eV}$, making the formation of interstitials almost as likely as that of the vacancies. As expected, the contribution of vdW interactions to the formation energy is much smaller than covalent bonding; nevertheless, vdW interactions are relevant in determining the relative stability of point defects. Overall, we note that our $\mathrm{HSE}+\mathrm{vdW}$ formation energies are in very good agreement with $G W$ calculations [21], the recent rs-LDA- 


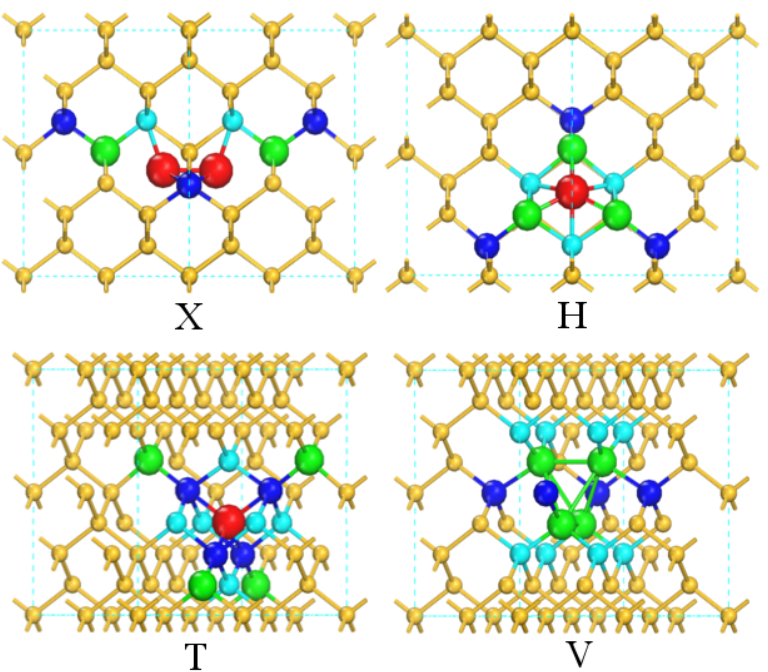

FIG. 1. (Color online) The crystal structure of the X, H, T, and $\mathrm{V}$ defects in Si. For V the most stable JT-distorted configuration is shown. The size of the balls indicates the change in the atomic vdW $C_{6}$ coefficients [39]. The $C_{6}$ coefficients increase with increasing size of the balls (in the order blue-green $<$ blue $<$ green $<$ red) .

TABLE II. Atomic vdW $C_{6}$ coefficients [39] (in hartree.bohr ${ }^{6}$ ) as a function of the atomic distance ( $L$ in $\AA$ ) from the defect center. $Z_{i}$ labels the $i$-th nearest neighbor $(x=0$ corresponds to the defect). For X, H, and T, a 64-atom cell was used. For $\mathrm{V}, 216$-atom cell was used. Calculations for $\mathrm{X}, \mathrm{H}$, and $\mathrm{T}$ defects in a larger 216 -atom supercell increase the $C_{6}$ values by less than 8 hartree.bohr ${ }^{6}$.

\begin{tabular}{cccccccc}
\hline \hline & & $Z_{0}$ & $Z_{1}$ & $Z_{2}$ & $Z_{3}$ & $Z_{4}$ & $Z_{5}$ \\
\hline $\mathrm{X}$ & $C_{6}$ & 322 & 170 & 186 & 198 & 186 & 161 \\
& $L$ & 0 & 2.33 & 2.48 & 2.82 & 5.08 & 6.01 \\
$\mathrm{H}$ & $C_{6}$ & 524 & 215 & 196 & 206 & 164 & 160 \\
& $L$ & 0 & 2.41 & 2.42 & 4.66 & 5.88 & 6.93 \\
$\mathrm{~T}$ & $C_{6}$ & 503 & 194 & 174 & 204 & 162 & 161 \\
& $L$ & 0 & 2.47 & 2.71 & 4.81 & 5.91 & 6.05 \\
$\mathrm{~V}$ & $C_{6}$ & - & 206 & 182 & 174 & 165 & 168 \\
& $L$ & 0 & 1.98 & 3.55 & 3.80 & 5.78 & 5.89 \\
Bulk $[27,29]$ & $C_{6}$ & & \multicolumn{7}{c}{168} \\
\hline \hline
\end{tabular}

RPA study [23], and experimental data for the interstitials (4.2-4.7 eV). Both our results and the rs-LDA-RPA values for the vacancy formation energy suggest a larger value than most of the experimental estimates (2.1-4.0 eV) $[3-5,7-12]$. QMC calculations yield formation energies of interstitials that are somewhat larger than those found with HSE+vdW, $G W$, and rs-LDA-RPA methods. However, QMC values are very sensitive to the employed calculation parameters, and exhibit variations of up to $0.3 \mathrm{eV}$ between different studies [16-18].

We then turn to the question of why the effect of $\mathrm{vdW}$ interactions on the formation energy is strongly dependent on the defect geometry. First, we reveal the sensitive dependence of polarization on the nature of the defect.

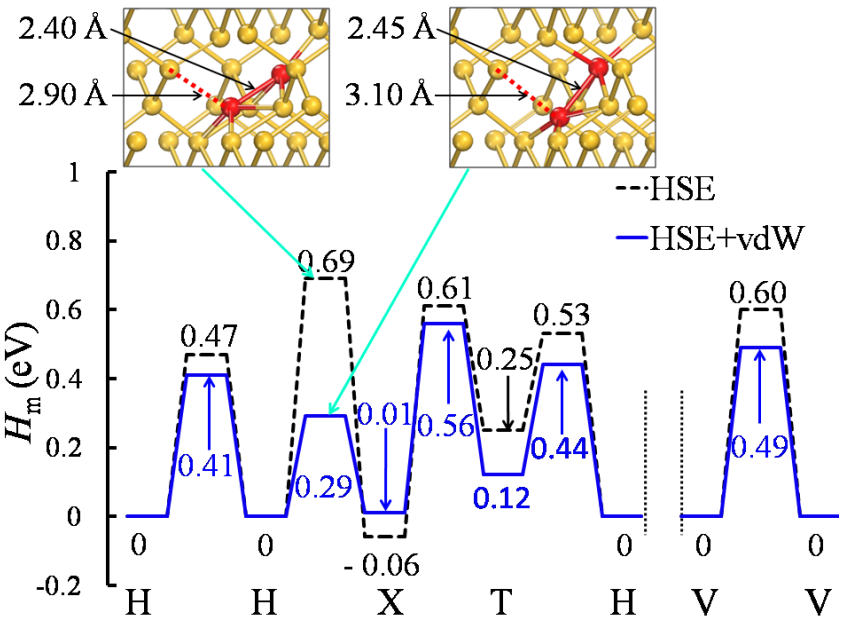

FIG. 2. Relative energies and migration barriers for different diffusion pathways of interstitials and vacancies in $\mathrm{Si}$. The insets show the geometry of the transition state along the $\mathrm{H}-\mathrm{X}$ pathway, determined with and without including vdW interactions.

Figure 1 illustrates the changes in the $\mathrm{vdW} C_{6}$ coefficients around defect sites for the four studied defects $(\mathrm{X}, \mathrm{H}, \mathrm{T}$, and $\mathrm{V}$ ) upon including the electrodynamic screening [29]. Table II shows the screened atomic $C_{6}$ coefficients [39] as a function of the atom distance from the defect center. In the case of interstitials, the $C_{6}$ coefficients of the atoms around the defect are significantly increased compared to the pristine Si bulk (168 hartree-bohr ${ }^{6}$ ) [27, 29]. It is noteworthy that the screened $C_{6}$ coefficients of the pristine Si bulk are in excellent agreement with those obtained using TDDFT. The polarization effects are relatively far reaching, extending as far as $5 \AA$ from the defect center. In addition, the polarization strongly depends on the nature of the defect, being roughly one-dimensional for the $\mathrm{X}$ interstitial, two-dimensional for the $\mathrm{H}$ interstitial, and three-dimensional for $\mathrm{T}$ and $\mathrm{V}$ defects. The static polarizability follows the same trend as the $C_{6}$ coefficients, indicating that the interstitials significantly influence the electrostatic screening in $\mathrm{Si}$ [40]. The dependence of polarization on the nature of the defect suggests an explanation for the observed trend in vdW interactions. The low-dimensional effect of $\mathrm{H}$ and $\mathrm{X}$ interstitials on the polarization leads to an almost negligible contribution from vdW interactions for these two defects, while a more pronounced vdW energy contribution for the T interstitial stems from a larger "vdW sphere" that encloses the most polarizable Si atoms. The formation of the vacancy is accompanied by a reduction in vdW interactions, since the loss of an atom from the Si bulk is not compensated by an appreciable gain in polarization around the vacancy site (see Table II).

In addition to the formation energy, an important ingredient to understand defect mobility is the migration 
TABLE III. Migration barrier of interstitials (Si-I), vacancies (Si-V), bond-centered hydrogen $\left(\mathrm{H}_{\mathrm{BC}}^{+}\right.$and $\left.\mathrm{H}_{\mathrm{BC}}\right)$, and oxygen in $\mathrm{Si}\left(\mathrm{O}_{\mathrm{i}}\right)$. All values are reported in $\mathrm{eV}$.

\begin{tabular}{cccccc}
\hline \hline & PBE & PBE+vdW & HSE & HSE+vdW & Exp. \\
\hline $\mathrm{Si}-\mathrm{I}$ & 0.40 & 0.25 & 0.47 & 0.29 & $0.20[41]$ \\
$\mathrm{Si}-\mathrm{V}$ & 0.51 & 0.39 & 0.60 & 0.49 & $0.45[42]$ \\
$\mathrm{H}_{\mathrm{BC}}^{+}$ & 0.54 & 0.40 & 0.72 & 0.58 & $0.48[43-$ \\
$\mathrm{H}_{\mathrm{BC}}$ & 0.42 & 0.28 & 0.52 & 0.39 & $45]$ \\
& & & & & $2.44-$ \\
$\mathrm{O}_{\mathrm{i}}$ & 2.23 & 2.03 & 2.92 & 2.73 & $2.56[46-$ \\
& & & & & $48]$ \\
\hline \hline
\end{tabular}

barrier. Here the theoretical situation is much less certain when compared with the defect formation energies, for which state-of-the-art first-principles methods are in good agreement as shown in Table I. To the best of our knowledge, direct calculations of migration barriers using $G W$ and QMC methods are not available. Figure 2 shows the energy diagram of migration barriers for interstitials and vacancies. In the case of interstitials, our HSE calculations reveal that the $\mathrm{H}-\mathrm{H}$ pathway is dominant with the migration barrier $H_{\mathrm{m}, \mathrm{H}-\mathrm{H}}=0.47 \mathrm{eV}\left(H_{\mathrm{m}, \mathrm{H}-\mathrm{X}}=\right.$ $0.75 \mathrm{eV}, H_{\mathrm{m}, \mathrm{H}-\mathrm{T}}=0.53 \mathrm{eV}$, and $\left.H_{\mathrm{m}, \mathrm{T}-\mathrm{X}}=0.67 \mathrm{eV}\right)$. In contrast to the defect formation energy, where HSE exhibits a good agreement with higher-level calculations and experimental values, there is a marked difference between HSE $(0.47 \mathrm{eV}-0.75 \mathrm{eV})$ and experiment $(0.20$ $\mathrm{eV}$ [41]) for the migration barrier height. Upon including the vdW interactions, all HSE migration barriers are decreased when using the HSE+vdW approach. In particular, the barrier of $\mathrm{H}-\mathrm{X}$ pathway is decreased from 0.75 $\mathrm{eV}$ to $0.29 \mathrm{eV}$. Notably, this value is in excellent agreement with the experimental value of $0.20 \mathrm{eV}$ measured at cryogenic temperatures $\left(-273 \sim-150{ }^{\circ} \mathrm{C}\right)$ [41]. To further elucidate the effect of $\mathrm{vdW}$ interactions on barrier heights, we calculated the diffusion of bond-centered hydrogen $\left(\mathrm{H}_{\mathrm{BC}}^{+}\right.$and $\left.\mathrm{H}_{\mathrm{BC}}\right)$ and oxygen in $\mathrm{Si}\left(\mathrm{O}_{\mathrm{i}}\right)$ (Table III). We find that vdW interactions decrease the migration barriers of these defects, which is analogous to the case of interstitials and vacancies. Importantly, the migration barriers of $\mathrm{H}_{\mathrm{BC}}^{+}$and $\mathrm{O}_{\mathrm{i}}$ by $\mathrm{HSE}+\mathrm{vdW}$ are also in agreement with experimental values [43-48]. Therefore, the decrease of defect migration barriers in semiconductors under the influence of $\mathrm{vdW}$ interactions seems to be a rather general phenomenon. One can see from the insets in Figure 2 that the transition state structure of $\mathrm{H}$ $\mathrm{X}$ pathway is substantially modified upon including the vdW energy, while those of other pathways are almost unchanged (not shown). The vdW interactions decrease the lattice constant of Si bulk by $0.02 \AA$, changing the configuration of the transition state ( $\mathrm{H}-\mathrm{X}$ pathway) by decreasing the interatomic distance between the defect atom and its nearest neighbors by as much as $0.2 \AA$. It is noteworthy that the exact exchange and vdW interac-
TABLE IV. Formation energy $(\mathrm{eV})$ of the vacancies for the heavier semiconductors and $\mathrm{Si}$ (the monovacancy $V$ in Ge, the As vacancy $V_{\mathrm{As}}$ in GaAs and InAs, and the $\mathrm{P}$ vacancy $V_{\mathrm{P}}$ in InP; multi-atom vacancies in $\mathrm{Si}$ ). The chemical potential $\mu$ has two limits for each of GaAs, InP and InAs. $\Delta$ indicates the fraction of $\mathrm{vdW}$ energy contribution to the formation energy.

\begin{tabular}{ccccc}
\hline \hline & $\mu$ & HSE & HSE+vdW & $\Delta$ \\
\hline Ge- $V$ & & 3.21 & 3.47 & $7.5 \%$ \\
GaAs- $V_{\text {As }}$ & As-rich & 3.96 & 4.23 & $6.4 \%$ \\
& Ga-rich & 3.01 & 3.23 & $6.8 \%$ \\
InP- $V_{\mathrm{P}}$ & In-rich & 3.12 & 3.33 & $6.3 \%$ \\
& P-rich & 2.28 & 2.49 & $8.4 \%$ \\
InAs- $V_{\text {As }}$ & In-rich & 3.04 & 3.31 & $8.2 \%$ \\
& As-rich & 2.15 & 2.42 & $11.2 \%$ \\
Si- $V_{2}$ & & 6.08 & 6.56 & $7.3 \%$ \\
Si- $V_{3}$ & & 8.45 & 9.20 & $8.2 \%$ \\
Si- $V_{4}$ & & 8.07 & 9.18 & $12.1 \%$ \\
Si- $V_{5}$ & & 9.32 & 10.61 & $12.2 \%$ \\
Si- $V_{6}$ & & 10.28 & 11.77 & $12.7 \%$ \\
\hline \hline
\end{tabular}

tions have an opposite effect on the migration barriers. In the case of $\mathrm{Si}$ vacancy, $\mathrm{HSE}+\mathrm{vdW}$ yields a migration barrier $H_{\mathrm{m}, \mathrm{V}-\mathrm{V}}=0.49 \mathrm{eV}$, which once again is in excellent agreement with the experimental value of $0.45 \mathrm{eV}$ at cryogenic temperatures [42].

We can use the HSE+vdW method to calculate the activation energy for defect diffusion in $\mathrm{Si}$, which is just the sum of the formation energy and the migration barrier. We find that the interstitial diffusion mechanism $\left(H_{\mathrm{A}, \mathrm{H}-\mathrm{X}}=4.69 \mathrm{eV}\right)$ is slightly preferred over the vacancy mechanism $\left(H_{\mathrm{A}, \mathrm{V}-\mathrm{V}}=4.87 \mathrm{eV}\right)$. These values are in good agreement with the proposed values $H_{\mathrm{A}, \mathrm{I}}=4.68$ $\mathrm{eV}$ and $H_{\mathrm{A}, \mathrm{V}}=4.86 \mathrm{eV}$ [10] based on the diffusion of $\mathrm{P}$ and $\mathrm{Sb}$ atoms in $\mathrm{Si}[9]$.

We also generalize our conclusions regarding the relevance of vdW interactions for studying defects in heavier semiconductors, including Ge, GaAs, InP, and InAs (Table IV). In these cases, vdW interactions are found to play an increasingly important role on the stability of the vacancy, increasing the formation energy by as much as $11.2 \%$ compared to $4.6 \%$ effect in $\mathrm{Si}$. This is due to the more polarizable nature of these semiconductors than Si. We also find that vdW interactions play a larger role for multi-atom vacancies and more complex defect structures in $\mathrm{Si}$, increasing the formation energy by $12.7 \%$ for hexavacancy $V_{6}\left(E_{\mathrm{vdW}}=1.49 \mathrm{eV}\right)$. These results provide an interesting avenue for future work.

In conclusion, the HSE+vdW method solves the underestimation of $\mathrm{PBE}$ on defect formation energies and the overestimation of HSE on defect migration barriers, yielding novel insights into the stability and mobility of point defects in semiconductors. Notably, our calculations successfully explain a series of experimental observations for the diffusion of interstitials, vacancies, and impurities in Si. These results suggest that HSE+vdW method can be employed for predictions 
in materials where the correct description of electronic structure (band gaps) and the long-range electron correlation is essential.

The authors acknowledge the European Research Council (ERC Starting Grant VDW-CMAT) for support. Part of this work was carried out at the Institute for Pure and Applied Mathematics (IPAM) at the University of California in Los Angeles (UCLA).

tkatchen@fhi-berlin.mpg.de

[1] A. M. Tyryshkin et al., Nat. Mater. 11, 143 (2012).

[2] D. Riedel et al., Phys. Rev. Lett. 109, 226402 (2012).

[3] S. Dannefaer, P. Mascher, and D. Kerr, Phys. Rev. Lett. 56, 2195 (1986).

[4] P. M. Fahey, P. B. Griffin, and J. D. Plummer, Rev. Mod. Phys. 61, 289 (1989).

[5] Y. Shimizu, M. Uematsu, and K. M. Itoh, Phys. Rev. Lett. 98, 095901 (2007).

[6] H. Bracht, H. H. Silvestri, I. D. Sharp, and E. E. Haller, Phys. Rev. B 75, 035211 (2007).

[7] H. Bracht, N. A. Stolwijk, and H. Mehrer, Phys. Rev. B 52, 16542 (1995).

[8] H. Bracht, E. E. Haller, and R. Clark-Phelps, Phys. Rev. Lett. 81, 393 (1998).

[9] A. Ural, P. B. Griffin, and J. D. Plummer, J. Appl. Phys. 85, 6440 (1999).

[10] A. Ural, P. B. Griffin, and J. D. Plummer, Phys. Rev. Lett. 83, 3454 (1999).

[11] H. Bracht et al., Phys. Rev. Lett. 91, 245502 (2003).

[12] V. Ranki and K. Saarinen, Phys. Rev. Lett. 93, 255502 (2004).

[13] R. Vaidyanathan, M. Y. L. Jung, and E. G. Seebauer, Phys. Rev. B 75, 195209 (2007).

[14] Y. Bar-Yam and J. D. Joannopoulos, Phys. Rev. B 30, 1844 (1984).

[15] P. E. Blöchl et al., Phys. Rev. Lett. 70, 2435 (1993).

[16] W.-K. Leung et al., Phys. Rev. Lett. 83, 2351 (1999).

[17] E. R. Batista et al., Phys. Rev. B 74, 121102 (2006).

[18] W. D. Parker, J. W. Wilkins, and R. G. Hennig, Phys. Status Solidi B 248, 267 (2011).

[19] C. G. Van de Walle and J. Neugebauer, J. Appl. Phys. 95, 3851 (2004).

[20] C. G. Van de Walle and A. Janotti, Phys. Status Solidi B 248, 19 (2011).

[21] P. Rinke, A. Janotti, M. Scheffler, and C. G. Van de Walle, Phys. Rev. Lett. 102, 026402 (2009).

[22] R. Ramprasad, H. Zhu, P. Rinke, and M. Scheffler, Phys. Rev. Lett. 108, 066404 (2012).

[23] F. Bruneval, Phys. Rev. Lett. 108, 256403 (2012).
[24] S. K. Estreicher, D. J. Backlund, C. Carbogno, and M. Scheffler, Angew. Chem. Int. Ed. 50, 10221 (2011).

[25] X. Ren, P. Rinke, C. Joas, and M. Scheffler, J. Mater. Sci. 47, 7447 (2012).

[26] N. Marom, A. Tkatchenko, M. Scheffler, and L. Kronik, J. Chem. Theory Comput. 6, 81 (2010).

[27] G. X. Zhang, A. Tkatchenko, J. Paier, H. Appel, and M. Scheffler, Phys. Rev. Lett. 107, 245501 (2011).

[28] J. Heyd, G. E. Scuseria, and M. Ernzerhof, J. Chem. Phys. 124, 219906 (2006).

[29] A. Tkatchenko, R. A. DiStasio, Jr., R. Car, and M. Scheffler, Phys. Rev. Lett. 108, 236402 (2012).

[30] T. Bučko, S. Lebègue, J. Hafner, and J. G. Ángyán, Phys. Rev. B 87, 064110 (2013).

[31] V. Voronkov, J. Cryst. Growth 59, 625 (1982).

[32] G. Mensing et al., J. Non-Cryst. Solids 299, 621 (2002).

[33] V. Blum et al., Comput. Phys. Commun. 180, 2175 (2009).

[34] X. Ren et al., New. J. Phys. 14, 053020 (2012).

[35] J. P. Perdew, K. Burke, and M. Ernzerhof, Phys. Rev. Lett. 77, 3865 (1996).

[36] See supplemental material at http:// for a detailed description of our DFT and DFT+vdW calculations.

[37] K. Sueoka, E. Kamiyama, H. Kariyazaki, and J. Vanhellemont, Phys. Status Solidi C 9, 1947 (2012).

[38] J. Klimeš and A. Michaelides, J. Chem. Phys. 137, 120901 (2012).

[39] We calculate the homoatomic $C_{6}$ coeffcient for an atom $i$ as the Casimir-Polder integral over projected polarizability, resulting from a sum over screening contributions from all atoms $j$ in the system, including the necessary periodic images. Such projection yields the exact total polarizability and $C_{6}$ coefficient of the complete system by construction. See Ref. [29] for more details.

[40] Currently used hybrid DFT functionals, such as HSE, employ a constant range-separation parameter. The correct treatment of systems with non-homogeneous polarization may require using position dependent dielectric screening.

[41] G. D. Watkins, in ECS Proc. 99, 38 (1999).

[42] G. D. Watkins, J. Phys. Soc. Jpn. Suppl. II 18, 22 (1963).

[43] A. Van Wieringen and N. Warmoltz, Physica 22, 849 (1956).

[44] C. H. Seager and R. A. Anderson, Appl. Phys. Lett. 53, 1181 (1988).

[45] Y. V. Gorelkinskii and N. N. Nevinnyi, Physica B 170, 155 (1991).

[46] J. W. Corbett, R. S. McDonald, and G. W. Watkins, J. Phys. Chem. Solids 25, 873 (1964).

[47] U. Gösele and T. Y. Tan, Appl. Phys. A 28, 79 (1982).

[48] F. Shimura, Oxygen in Silicon (Academic, San Diego, 1994). 